\title{
Optimal Meter Placement by Reconciliation Conventional Measurements and Phasor Measurement Units (PMUs)
}

Reza Kaihani

Shiraz University, Iran, Seifi@shirazu.ac.ir

Ali Reza Seifi

Shiraz University, Iran, rkaihani367@yahoo.com

Follow this and additional works at: http://digitalcommons.wayne.edu/jmasm

Part of the Applied Statistics Commons, Social and Behavioral Sciences Commons, and the Statistical Theory Commons

\section{Recommended Citation}

Kaihani, Reza and Seifi, Ali Reza (2010) "Optimal Meter Placement by Reconciliation Conventional Measurements and Phasor Measurement Units (PMUs)," Journal of Modern Applied Statistical Methods: Vol. 9 : Iss. 1 , Article 29.

DOI: $10.22237 /$ jmasm/1272688080

Available at: http://digitalcommons.wayne.edu/jmasm/vol9/iss1/29 


\title{
Optimal Meter Placement by Reconciliation Conventional Measurements and Phasor Measurement Units (PMUs)
}

\author{
Reza Kaihani Ali Reza Seifi \\ Shiraz University, \\ Iran
}

The success of state estimation depends on the number, type and location of the established meters and RTUs on the system. A new method by incorporating conventional measurements and New Technology of Phasor Measurement Units (PMU) is proposed. Conventional meters (power injection and power flow measurements) are allocated in order to reduce the number of meters, RTUs, critical measurements, critical sets and leverage points, and also to improve the numerical stability of equations; a genetic algorithm is used for optimization. A second step involves adding PMUs in areas in which it is expected that the accuracy of state estimation will be improved.

Key words: State estimation, meter placement, network observability, Phasor Measurement Units, PMU, leverage points.

Introduction

Current energy management systems (EMSs) must accurately monitor power system state variables, i.e. the voltage phasors (voltages in module and phase) of each bus in real time. The primary monitoring tool is the state estimation (SE), which constitutes the core of all control operations. Installing a new state estimator or upgrading an existing one requires - among other considerations - evaluation of the metering configuration. Determination of the best possible combination of meters for monitoring a given power system is referred to as the optimal meter placement problem. Fundamentally, the metering scheme must provide enough information to allow power system state estimation. Planning metering systems for power system monitoring is a complex task, not only due to the problem dimension itself (number of possible configurations), but also to the need of

Ali Reza Seifi is an Associate Professor in the School of Electrical and Computer Engineering. Email: seifi@shirazu.ac.ir. Reza Kaihani is a Msc student in the Department of power and control Engineering in the School of Electrical and Computer Engineering. Email: rkaihani367@yahoo.com. establishing a trade-off between SE performance and metering system costs. With an adequate metering system, the SE can successfully process the available information and obtain reliable estimates of system operating conditions, which can then be used for further analyzes and for control actions.

In the design of measurement point locations, first it should be considered that the measurement system must satisfy the basic condition of state estimation: observability of the network. In addition to this essential prerequisite, it is also necessary to consider other issues such as accuracy, reliability and economy. Network observability analysis determines whether the network is observable or not by the type and placement of the measurements; the topology of network observability is related to the type and placement of the measurements.

Several methods of network observability analysis, such as, numerical (Abur \& Expósito, 2004; Monticelli \& Wu, 1986) and topological (Abur \& Expósito, 2004; Krumpholz, Clements \& Davis, 1980) have been introduced to determine if the network is observable or island observable. Implementation of synchronized phasor measurements presents 


\section{SEIFI \& KAIHANI}

an opportunity for improvements in power system state estimation.

As an addition to standard real and reactive power and voltage and current magnitude measurements, the Phasor Measurement Unit (PMU) provides voltage and current phasor measurements $\left(\mathrm{Ve}^{\mathrm{i} \theta}, \mathrm{Ie}^{\mathrm{j} \theta}\right)$. PMUs provide positive sequence value measurements, are available from 20 times per cycle and can be synchronized with measurements obtained from another PMU. The PMUs are accurate and can take measurements synchronously, thus improving the performance of state estimation.

Several research articles have been published regarding algorithms developed with the objective of attaining optimal measurement plans for power system state estimation, taking into account some of the previously described requirements. Cost of installing new meters and reduction of the number of critical p-set measurements are taken into consideration in the formulations of (Souza, et al., 2005; Mori \& Iida, 1993; Riccieri \& Falcão, 1999; Mori \& Matsuzaki, 1999; Allemong, Radu \& Sasson, 1982; Antonio, Torreão \& Filho, 2001). Accuracy of the weighted least squares state estimation for the chosen measurement design is also used as one of the objectives in these studies (Mori \& Iida, 1993). In Monticelli and $\mathrm{Wu}$ (1986) and Magnago and Abur (2000) a metering system was designed for a basic network and possible occurrence of topology changes and/or measurement losses. Pioneering work in PMU development and utilization has been accomplished by Phadke, et al. (1986). For details on PMU placement problems in power systems, see Zovanocic \& Cairns, 1996; Milosevic \& Begovic, 2003; and Rice \& Heydt, 2006.

In this article planning of measurement systems is implemented in two steps. The first step uses conventional meters, power injection measurement and power flow measurement in pairs unit $(\mathrm{P}, \mathrm{Q})$ to achieve a primary outline. The objective function in this step is observability, reducing the cost of meters and RTU, decreasing critical measurements and critical sets, minimizing the number of leverage points and improving numerical stability. The second step adds PMUs to the primary design from step 1 to improve the accuracy state estimation and to speed up convergence. That detection of bad data in bad leverage points due to errors or malfunctions in meters is not simple and the number of leverage points is reduced to avoid this situation; as is known, however, the existence of good leverage points (free of bad data) causes the accuracy of state estimation to increase (Abur \& Expósito, 2004). To avoid losing the positive qualification by adding PMUs, in step 2 the accuracy will be increased to compensate for the absence of leverage points in the pre-designed measurement system developed in step 1 .

\section{Linear State Estimation}

The conventional method for power system state estimation is the weighted least squares (WLS) state estimation (Abur \& Expósito, 2004). The WLS state estimator equations relating to the measurements and the state vector are:

$$
z=H \cdot x+e
$$

where $\mathrm{x}$ and $\mathrm{z}$ are the $\mathrm{n} \times 1$ state and $\mathrm{m} \times 1$ measurement vectors; $\mathrm{H}$ is the $m \times n$ Jacobian matrix, $\mathbf{e}$ is the $m \times 1$ measurement error vector, $m$ is the number of measurements and $n$ is the number of buses. The SE can be formulated as weighted least-squares (WLS) problem

$$
\min J(x)=[z-H x] R^{-1}[z-H \hat{x}]
$$

The state estimate $\hat{x}$ by minimization $\mathrm{J}(\mathrm{x})$ in [ ] can be obtained through the WLS method by satisfying the following Optimality condition:

$$
\begin{aligned}
& \frac{\partial J(x)}{\partial x}=H^{T} R^{-1}[z-H \hat{x}]=0 \\
& \hat{x}=G^{-1} H^{T} R^{-1} z
\end{aligned}
$$

where $\mathrm{G}=\mathrm{H}^{T} \mathrm{R}^{-1} \mathrm{H}$ is known as the gain matrix. 


\section{Observability Analysis}

Observability analysis is a search process for portions of a power network; given the network and measurement topology, state estimation can be performed. Usually, the linearized and decoupled state estimator is adopted to perform observability analysis. Hereafter, for simplicity, the $\mathrm{Pe}$ (active powerangle) model will be used. A system is said to be observable if the gain matrix is nonsingular, which can be verified during its triangular factorization (no zero pivots, if the reference bus angle is not included) (see Abur \& Expósito, 2004; Monticelli \& Wu, 1986; Krumpholz, Clements \& Davis, 1980).

\section{Condition Number}

The condition number of a nonsingular square matrix $\mathrm{A}$ is defined as:

$$
\mathrm{K}_{\mathrm{G}}=\|\mathrm{A}\| \cdot\left\|\mathrm{A}^{-1}\right\|
$$

where $\|. .$.$\| represents a matrix norm (Abur \&$ Expósito, 2004; Rice \& Heydt, 2007; Reza \& Ross, 2001). If 2-norm is used, the condition number can be calculated using

$$
\mathrm{K}_{\mathrm{G}}=\frac{\lambda_{\mathrm{s}}}{\lambda_{1}}
$$

where $\lambda$ denotes the eigenvalues of $A$ respectively, subscript $s$ refers to the largest eigenvalues and subscript 1 refers to the smallest values. The condition number is equal to unity for identity matrices and tends to infinity for matrices approaching singularity. A large condition number in value is indicative of an illconditioned matrix (Abur \& Expósito, 2004).

In state estimation, the sensitivity of the estimate of $\mathrm{x}$ to noise is improved (lessened) when $\mathrm{KG}$ (the condition number of gain matrix) is small, and the sensitivity is worsened (increased) when KG is large. Typical threshold values of $\mathrm{KG}$ in state estimation applications, beyond which designers of a state estimator become concerned, are approximately 105 (Rice \& Heydt, 2007).

\section{Leverage Points}

Some measurements of a power system may have a much stronger influence on the state estimate than others due to their location, the local measurement redundancy, the network topology and parameters. These points are outliers in the space spanned by the row vectors of the Jacobian matrix, meaning that they do not follow the pattern of the point cloud in that space. Such measurements, referred to as leverage measurements, will distort the solution of the least absolute value estimation when they carry bad data.

Two cases are associated with leverage points. When a measurement is a leverage point and has a wrong metered value, it is a bad leverage point; identification of the bad measurement becomes very difficult by conventional methods. Residual covariance for these measurements will be numerically insignificant. If, however, the measurement is a leverage point and has a good metered value, it is a good leverage point and heavily reinforces the M-estimator's performance.

\section{Projection Statistics}

A robust measure of leveraging the effect of a measurement was applied to the power system state estimation by Mili, et al., 1996; this measure is called the projection statistic (PSi) and is defined for a measurement $\mathrm{i}$ as

$$
P S_{i}=\max \frac{\left|H_{i}^{t} \cdot H_{k}\right|}{\beta} \quad \text { for } k=1,2, \ldots, m
$$

where

$$
\begin{aligned}
& \beta=1.926 \text { lomed }_{\mathrm{i}}\left\{\operatorname{lomed}_{\mathrm{j} \neq \mathrm{i}}\left\{\left|\mathrm{H}_{\mathrm{i}}^{\mathrm{T}} \cdot \mathrm{H}_{\mathrm{k}}+\mathrm{H}_{\mathrm{j}}^{\mathrm{T}} \cdot \mathrm{H}_{\mathrm{k}}\right|\right\}\right\} \\
& \quad 1 \leq \mathrm{i}, \mathrm{j}, \mathrm{k} \leq \mathrm{m}
\end{aligned}
$$

and $\operatorname{lomed}_{\mathrm{i}}\{\mathrm{x}\}$ is the low median of the $\mathrm{m}$ number in $\mathrm{x}=\{\mathrm{x} 1, \mathrm{x} 2, \ldots, \mathrm{xm}\}$.

The projection statistic PSi can be shown to behave approximately like a Chisquare random variable. Measurement $i$ is the related to the sparsity structure of the row $\mathrm{H}_{\mathrm{i}}$. Hence, measurement $\mathrm{i}$ is identified as a leverage point if $P S_{i}>\chi_{k, 0.975}^{2}$ where, $\mathrm{k}$ is the number of 


\section{SEIFI \& KAIHANI}

nonzero entries in the row $\mathrm{H}_{\mathrm{i}}$ of the measurement Jacobian $\mathrm{H}$.

\section{Classification of Measurements}

Critical Measurement

Critical measurement is one whose elimination from the measurement will result in an unobservable system. The residual and standard deviation associated with a critical measurement always equals zero.

$$
\begin{aligned}
r(i) & =z(i)-\hat{z}(i) \\
\sigma_{E}(i) & =\sqrt{E(i, i)}=0
\end{aligned}
$$

where

$$
\begin{aligned}
& E=R-H G^{-1} H^{T} \\
& \sigma_{E}(i)=\sqrt{E(i, i)}
\end{aligned}
$$

and

$$
\hat{z}=H \hat{x}
$$

Redundant Measurement

A redundant measurement is a measurement that is not critical. Only redundant measurements may have nonzero residuals.

\section{Critical Set}

A critical set (Cset) is defined as a group of measurements (non-critical) in which the removal of any of such measurements makes the remaining of the group critical. Normalized residuals of measurements pertaining to a critical set (Cset) are equal and their correlation coefficients present maximum values. Suppose that measurements $i$ and $j$ belong to the same critical set, it follows that:

$$
\begin{aligned}
& \rho_{i j}=\frac{r_{N}(i)}{r_{N}(j)}=1 \\
& \gamma_{i j}=\frac{E(i, j)}{\sqrt{E(i, i)} \sqrt{E(j, j)}}=1
\end{aligned}
$$

This article employs a method detailed in Filho, et al., 2001 to detect critical measurements and sets using equations presented.
Genetic Algorithm

A genetic algorithm (GA) stems from an analogy of the Natural selection process. The GA has the following advantages:

(i) It is expected that the GA is capable of evaluating the global minimum; the GA is based on the multi-point search and does not get stuck with local minima; and

(ii) It is not necessary that the objective function is differentiable, that is, the objective function is arbitrary.

The GA evaluates the optimal solution to maximize the objective function called the fitness. Using the genetic operators such as crossover, mutation, and reproduction the optimal solution is searched to maximize the fitness. In this article, a GA is used to determine the optimal solution for redundant measurements for static state estimation. The specified values of the load flow calculation are taken as the basic measurements, the GA was archived in step 1 for designing the primary outline of the metering system and the measurement set is assumed to contain only the conventional measurements such as, power injections and power flows.

\section{Methodology}

Step 1: Metering System Design

To reduce the number (cost) of meters and RTUs, to abate critical measurement and critical set and leverage points and to decrease condition numbers, it is necessary that the SE equation converge rapidly and avoid illconditioned cases type of measurement. In step 1, power injection measurements and power flow measurements are used. The random measurement error standard deviation is:

$$
\sqrt{R}_{i}=\frac{\left(0.02 m+0.005 f_{s}\right)}{3}
$$

where $m=\sqrt{\mathrm{P}_{\mathrm{i}}^{2}+\mathrm{Q}_{\mathrm{i}}^{2}}$ is the true measurement value, and $f_{s}$ is full scale value. 
Fitness Function

To comply with requirements discussed a fitness function is proposed as:

$$
\begin{aligned}
F F= & \text { Nmeas }+k_{1} \times N r t u \\
& +k_{2} \\
& +k_{3} \times N l e p o+k_{4} \times N s c r+k_{5} \times N p c r
\end{aligned}
$$

where FF is the fitness function, Nmeas is the number of measurements, Nrtu is the number of RTUs, Nlepo is the number of leverage points, Nscr is the number of critical measurements, Npcr is the number of critical 2-set and k1, k2, $\mathrm{k} 3, \mathrm{k} 3, \mathrm{k} 4, \mathrm{k} 5$ are constants.

\section{Step 2: Addition of Voltage Phase Angle} Measurement

The addition of a voltage phase angle measurement to a conventional state estimator could greatly increase the accuracy of the state estimator if implemented correctly. In this step, adding PMUs to the pre-designed metering system developed in step 1 will increase accuracy.

\section{PMU Placement}

PMU placement can be accomplished via several different criteria including security concerns, observability and improvement in state estimation. Here the criterion used to determine the location of PMUs will be improvements in the state estimator performance. It is possible to examine the deviation of $\mathrm{x}^{\wedge}$ from the exact value of $\mathrm{x}$. Typically this comparison is not possible, however, due to the use of test beds with a known solution, it is possible to use normalized error, NE, to assess the accuracy of $\hat{x}$ with

$$
\mathrm{NE}=\frac{\left\|\mathrm{x}_{\text {exact }}-\hat{\mathrm{x}}\right\|_{2}}{\left\|\mathrm{x}_{\text {exact }}\right\|_{2}}
$$

To allocate the PMUs, first, the residual vector of $\operatorname{states}\left(\mathrm{x}_{\text {exact }}-\hat{\mathrm{x}}\right)$ is calculated, followed by the difference of the residual vector to mean. The greatest number in this vector will be the best candidate for installation as the first PMU.
By this method, the addition of PMUs to the measurement set will be continued until changes in NE are not observed. (See Milosevic \& Begovic, 2003 for details.)

Step 1

\section{Results}

The meter placement problem was modeled through GAs considering a binary encoding system in which each individual (chromosome) of a population corresponds to a proposed solution for the problem (metering system). A chromosome is represented by a vector whose elements are associated with meter types and locations. The chromosome dimension then corresponds to the maximum number of meters that can be installed in a given network (twice the number of branches plus the number of buses). The chromosome elements (genes) assume binary values and will be equal to 1 if a meter is placed and equal to 0 otherwise. It is assumed that all the power measurements are in active-reactive pairs; therefore, a single gene represents a pair of measurements. (The proposed method is applied to analyze the measurement placement plan of the IEEE-14 bus power system shown in Figure 1.)

During the search procedure, different values for GA parameters (crossover probability, mutation rate, and population size) were tested. The search process stopping criterion was based on a previously defined maximum number of generations. The genetic algorithm parameters used in the Step 1 to run the search for the optimal set of measurements are as follows:

- Maximum generation $=200$

- Population size $=100$

- $\quad$ Crossover probability $=0.7$

- Mutation probability $=0.01$

- Constants in FF are: $\mathrm{k} 1=20, \mathrm{k} 3=100, \mathrm{k} 4$ $=10^{4}$ and $\mathrm{k} 5=100$.

$k_{2}= \begin{cases}0 & \text { if condition number } \leq 1,000 \\ 10^{2} & \text { if } 1,000 \mid<\text { condition number }<100,000 \\ 10^{6} & \text { if condition number } \geq 100,000\end{cases}$

The IEEE- 14 bus system example with its measurement configuration shown in Figure 1 illustrates the proposed method (Step 1). Five 


\section{SEIFI \& KAIHANI}

injections measurements are located at buses 1 , $3,7,10,13$ and 16, and line flow measurements on lines 1-2, 1-5, 3-2, 3-4, 7-4, 6-5, 6-11, 6-12, $13-6,7-8,7-9,9-10,9-14,10-11,13-12$ and 1314. The evolution of the fitness for the best individual in each generation is presented in Figure 2.
Step 2

The procedure in Step 1 will be achieved to determine the optimal placement and number of added measurements (PMUs) to increase the accuracy of state estimation and to decrease the condition number of the gain matrix. In addition, it is assumed that PMUs are

Figure 1: IEEE 14-Bus System with Measurements

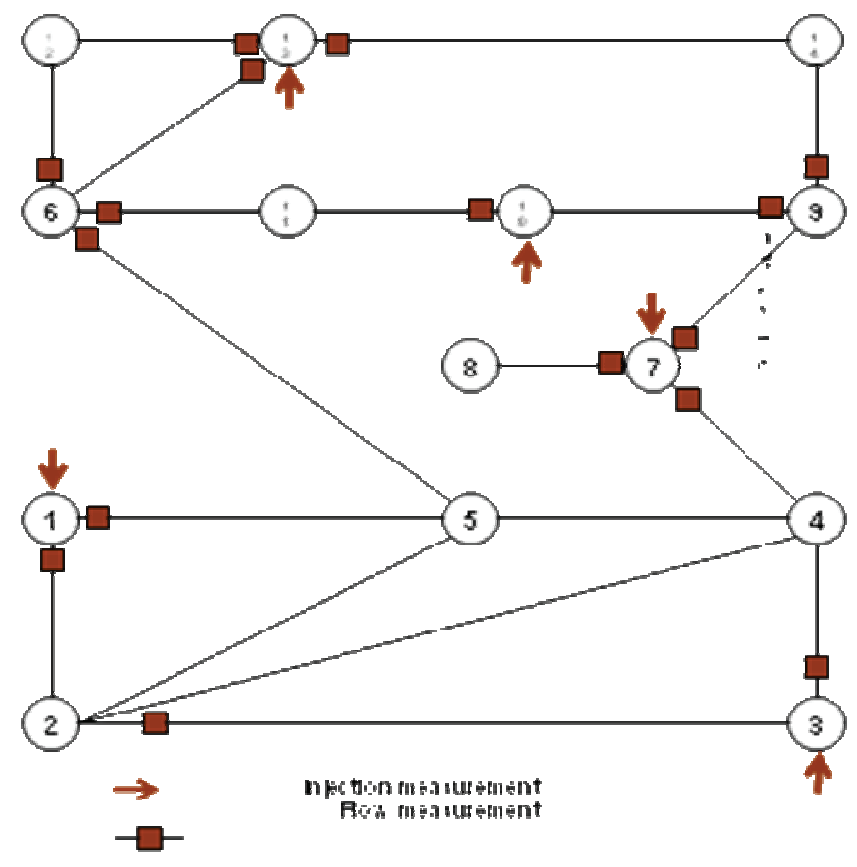

Figure 2: Convergence Characteristic of Best Solution (Step 1)

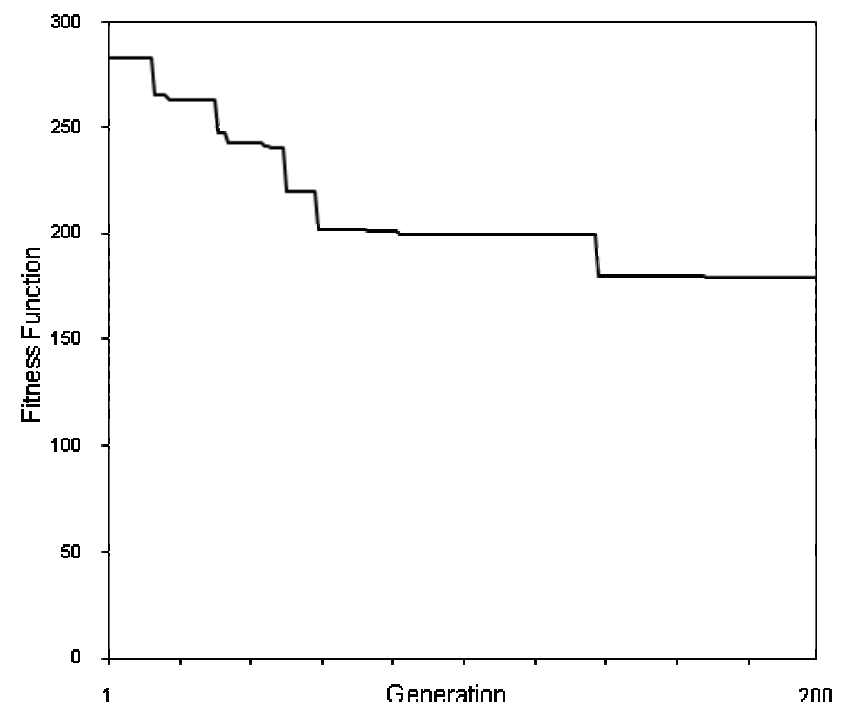


added to voltage phase angle measurements. The measurement standard deviation of PMUs is assumed to be 0.002 (radian).

Table 1 shows the results of the simulation for normalized errors when PMUs are added by the method discussed herein. An improvement in state estimation accuracy was distinguished as PMUs were added to System. Figure 3 shows the condition number variation along with the PMU amount with the increase of PMU amount, the scale of the estimator and the condition number of the equation became smaller. Thus, the model can improve the numerical stability of the SE equations.

\section{Conclusion}

An optimization problem has been formulated to determine the optimal design principle for arranging measurements where a number of metering systems should be minimized while some performance requirements should be observed. A genetic algorithm was applied to solve the optimal meter placement problem. Test results with the IEEE 14 bus system show that the proposed methodology is capable of obtaining optimal metering systems. Further the metering system was reinforced by adding PMUs to the system designed and the simulation shows that the new model can improve accuracy, the SE equations numerical stability and the convergence speed.

Table 1: Change in Normalized Error due to Install PMUs

\begin{tabular}{|c|c|c|}
\hline Location of PMUs & Normalized Error & Change in Normalized Error (\%) \\
\hline (No PMU) & 0.0015755 & \\
\hline 12 & 0.00031086 & -80.2691 \\
\hline 6,12 & 0.000055957 & -81.9993 \\
\hline $6,11,12$ & 0.000046249 & -17.3490 \\
\hline $6,11,12,13$ & 0.000040274 & -12.9191 \\
\hline $6,11,12,13,14$ & 0.000040091 & -0.4544 \\
\hline
\end{tabular}

Figure 3: Change in Condition Number

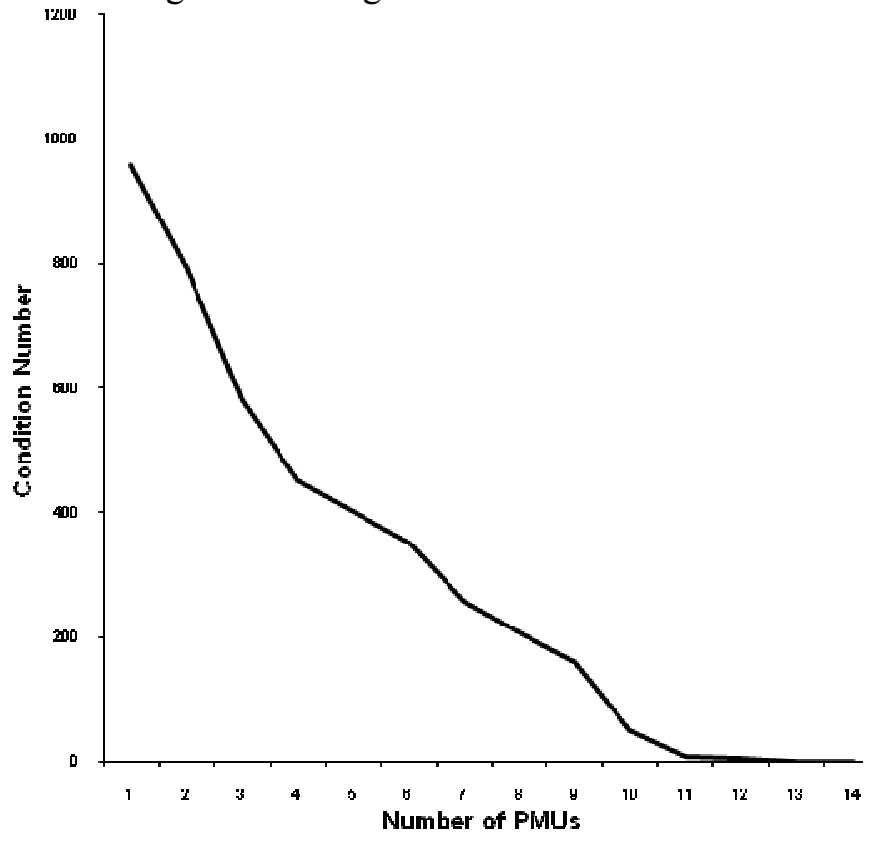




\section{SEIFI \& KAIHANI}

\section{References}

Abur, A., \& Expósito, A.G. (2004). Power system state estimation: theory and implementation. New York: Marcel Decker.

Monticelli, A., \& Wu, F. F. (1986).

Network observability: Identification of observable islands and measurement placement. IEEE Transactions on.PAS, PAS-104(5), 10351041.

Krumpholz, G. R., Clements, C. A., \& Davis, P. W. (1980). Power system observability: A practical algorithm using network topology. IEEE Trans on Power Apparatus and Systems, PAS-99(4), 1534-1542.

Souza, J. C. S., Do Coutto Filho, M. B, Meza, E. M., \& Schilling, M. T. (2005). Optimal metering systems for monitoring power networks under multiple topological scenarios. IEEE Trans on Power Apparatus and Systems, 20(4), 1700-1708.

Mori, H., \& Iida, S. (1993). Application of a genetic algorithm to meter allocation in electric power systems. Presentation at International Conference on Neural Networks.

Riccieri, O. F., \& Falcão, D. M. (1999). A meter placement method for state estimation using genetic algorithms. Intelligent System Application to Power Systems (ISAP), April, 360-364.

Mori, H., \& Matsuzaki, O. (1999). A tabu search based approach to meter placement in static state estimation. Intelligent System Application to Power Systems (ISAP), April, 365-369.

Allemong, J. J., Radu, I., \& Sasson, A. M. (1982). A fast and reliable state estimation algorithm for AEP's new control center. IEEE Transactions on Power Apparatus and Systems, 101(4), 933-944.

Antonio, A. B., Torreão, J. R. A., \& Do Coutto Filho, M. B. (2001). Meter placement for power system state estimation using simulated annealing. Proceedings of IEEE Porto PowerTech Conference, Porto, 146.
Magnago, F. H., \& Abur, A. (2000). A unified approach to robust meter placement against loss of measurements and branch outages. IEEE Trans on Power Apparatus and Systems, PWRS-15, 945-949.

Phadke, A.G., et al. (1986). State estimation with phasor measurements. IEEE Transactions on Power Systems, PWRS-1(1), 233-241.

Zovanocic, R., \& Cairns, C. (1996). Implementation of PMU technology in state estimation: An overview. IEEE AFRICON 4th, 2, 1006-1011.

Milosevic, B., \& Begovic, M.: No dominated sorting genetic algorithm for optimal phasor measurement placement. IEEE Transactions on Power Systems, 18(1), 69-75.

Rice, M. J., \& Heydt, G. T. (2006). Power system state estimation accuracy enhancement through the use of PMU measurements. IEEE/PES Transmission and Distribution Conference and Exhibition, 161165.

Rice, M. J., \& Heydt, G. T. (2007). Condition indicator analysis for the enhancement of power system. Report for PSERC Project, unpublished Project-Related Ph.D. Thesis.

Ebrahimian, R., \& Baldick, R. (2001). State estimator condition number analysis. IEEE Transactions on Power Systems, 16(2), 273-279.

Mili, L., Cheniae, M. G., Vichare, N. S., \& Rousseeuw, P. J. (1996). Robust state estimation based on projection statistics. IEEE Transactions on Power Systems, 11, 1118- 1127.

Do Coutto Filho, M. D., Souza, J. C. S., Oliveira, F. M. F., \& Schilling, M. T. (2001). Identifying critical measurements \& sets for power system state estimation. Proceedings of the IEEE Porto PowerTech Conference, September 2001. 